\title{
Sensitivity to phase distortions in central and peripheral vision
}

\author{
LEWIS O. HARVEY, JR., INGO RENTSCHLER, and CHRISTIAN WEISS \\ Institut für Medizinische Psychologie der Ludwig-Maximilians-Universität München \\ Munich, Federal Republic of Germany
}

\begin{abstract}
Sensitivity to phase quantization of a two-dimensional random texture pattern was investigated in central and peripheral vision, using the method of Caelli and Bevan (1982). The random texture was phase quantized with two-octave-wide bands centered on spatial frequencies of $2,4,8$, and 16 cycles per degree (cpd). For each of these bands, six modified versions of the original texture were prepared using different degrees of phase quantization: 24 steps of $15^{\circ}, 8$ steps of $45^{\circ}$, 5 steps of $72^{\circ}, 4$ steps of $90^{\circ}, 3$ steps of $120^{\circ}$, and 2 steps of $180^{\circ}$. The task of the subject was to discriminate between the original texture and each of the six phase-distorted versions. The amount of stimulus contrast required for threshold discrimination of each stimulus pair was determined using a two-alternative, forced-choice paradigm combined with a maximum-likelihood staircase procedure. Stimuli were presented sequentially at exposure durations of 1,000 and 125 msec. Subjects viewed the stimuli with foveal vision or $2^{\circ}$ in the right peripheral visual field. The results show that maximum sensitivity to phase quantization is found in the 4 cpd spatial frequency band and that sensitivity is greatly reduced with peripheral vision. Sensitivity to phase quantization is higher with 1,000- than with 125 -msec exposure duration.
\end{abstract}

In 1857 Aubert and Förster noted that targets seen in the peripheral visual field lack the quality of form in a way that cannot be understood in terms of reduced resolution or contrast. Subsequent research on peripheral visual function has concentrated on the measurement of acuity (Wertheim, 1894), luminance increment threshold (Aulhorn \& Harms, 1972; Harvey \& Pöppel, 1972; Pöppel \& Harvey, 1973), and grating contrast sensitivity (Daitch \& Green, 1969; Hilz \& Cavonius, 1974; Kelly, 1984a, 1984b; Rovamo \& Virsu, 1979; Virsu \& Rovamo, 1979). These visual functions have been shown to decrease as one moves from the fovea to the peripheral visual field.

Yet these findings do not explain Aubert and Förster's original observation. It has recently been suggested that peripheral vision may ignore phase relationships between spatial frequency components in complex visual patterns (Braddick, 1981; Hilz, Rentschler, \& Brettel, 1981; Julesz, 1981). A dependence of the visual quality of form upon phase information would seem plausible, since phase coding captures the relative position of the spatial frequency components in an image. Indeed, phase disturbances clearly alter image appearance in suprathreshold vision (Brettel, Caelli, Hilz, \& Rentschler, 1982), whereas phase information, at least under some conditions, may

\footnotetext{
Requests for reprints and correspondence to the first author should be addressed to: Lewis O. Harvey, Jr., Department of Psychology, Campus Box 345, University of Colorado, Boulder, CO 80309. This research was supported by a grant to I. Rentschler from the FraunhoferGesellschaft (InSan I 0784-V-6385) and by a Forschungsstipendium to L. Harvey from the Alexander von Humboldt Stiftung. The figures were prepared with graphics plotting equipment provided by Grant RR0701378 from the Biomedical Research Support Grant Program, National Institutes of Health.
}

not influence the detection of stimuli (Graham, 1972; Graham \& Nachmias, 1971; Nachmias \& Weber, 1975). The importance of phase is also found in a wide variety of signal types other than visual (Oppenheim \& Lim, 1981).

These considerations have motivated a new approach to the study of visual pattern discrimination. Burr (1980) and Lawden (1983) measured the discrimination of compound gratings which differed in the phase relationships of their grating components but had identical power spectra. Another technique for studying phase relationships has been introduced by Caelli and Bevan (1982). In this technique, phase-quantized versions of original images are prepared and subjects' ability to discriminate these from the originals is measured. A phase-quantized image is one in which the phase values of each spatial frequency component, which normally are distributed continuously across all values from 0 to $360^{\circ}$, are forced to a few discrete values (in $15^{\circ}$ steps, for example). Both of these techniques allow the measuring of visual sensitivity to image distortions under constant energy conditions. The phase quantization method is easier to apply to twodimensional images and the results are therefore more readily applied to the understanding of processing of "natural" images which contain a wide band of spatial frequencies at many orientations. We decided to use the phase-quantization paradigm of Caelli and Bevan (1982) to investigate sensitivity to phase distortion in peripheral vision.

\section{METHOD}

\section{Stimuli}

The stimuli were random checkerboard patterns of the type used by Caelli and Bevan (1982). A prototype random checkerboard pat- 
tern, $128 \times 128$ pixels large with a check size of $16 \times 16$ pixels, was generated. This prototype was identical to Texture $T_{3}$ used by Caelli and Bevan (1982, Figure 2). Additional stimuli were derived from this checkerboard pattern by first calculating its twodimensional discrete Fourier transform using the Cooley-Tukey fast Fourier transform (FFT) (Brigham, 1974) and then quantizing the phase in one of four frequency bands: (1) 1.0 to 4.0 cycles per degree (cpd), centered on $2.0 \mathrm{cpd}$; (2) 2.0 to $8.0 \mathrm{cpd}$, centered on $4.0 \mathrm{cpd}$; (3) 4.0 to $16.0 \mathrm{cpd}$, centered on $8.0 \mathrm{cpd}$; and (4) 8.0 to $32.0 \mathrm{cpd}$, centered on $16.0 \mathrm{cpd}$. For each of these four frequency bands, six modified stimuli were derived, based on six different levels of phase distortion. This distortion was based on six levels of phase quantization: (1) 24 steps of $15^{\circ}$; (2) 8 steps of $45^{\circ}$; (3) 5 steps of $72^{\circ}$; (4) 4 steps of $90^{\circ}$; (5) 3 steps of $120^{\circ}$; and (6) 2 steps of $180^{\circ}$. During this phase quantization, the phase of each spatial frequency component was transformed to the closest step value, without changing the amplitude of the component. For example, a phase angle of $13^{\circ}$ in the prototype stimulus would have been changed to $15^{\circ}$ in the $15^{\circ}$-step stimulus, but would have been changed to $0^{\circ}$ in the $45^{\circ}$-step stimulus. Finally, the stimuli were created by calculating the inverse two-dimensional Fourier transform of the phase-quantized spectra. It should be noted that the process of phase quantization changes the contrast of the stimuli somewhat, generally increasing contrast at the larger steps of quantization. This effect is independent of the spatial frequency band used for the quantization. The contrast values reported in the result section are the actual contrasts of the individual stimuli.

The stimuli were generated digitally by a Digital Equipment Corporation LSI 11/23 computer and were stored on disk. They were displayed using a Videograph digital image display system having a $512 \times 512$ pixel capacity and a 6 -bit gray scale (64 gray levels), and a Grundig GB45 black-and-white video monitor. At the viewing distance of $2.5 \mathrm{~m}$, each texture stimulus subtended $2^{\circ}$ of visual angle. The mean luminance of each stimulus was $60 \mathrm{~cd} / \mathrm{m}^{2}$; stimuli were viewed against a background luminance of $5 \mathrm{~cd} / \mathrm{m}^{2}$.

\section{Subjects}

The two subjects were two of the authors of this paper: C.W., a 28-year-old male, and L.H., a 41-year-old male. Both had normal or corrected-to-normal vision.

\section{Procedure}

The testing method was designed to measure the amount of stimulus contrast required to allow the subject to just discriminate between the original texture stimulus and each of the phase-distorted versions of that stimulus. This contrast level, referred to here as "threshold" contrast, is the contrast giving a 0.82 probability of correct discrimination in a two-alternative, forced-choice paradigm. This probability level, rather than the more conventional level of 0.75 , was used because the maximum likelihood staircase method we used (described in more detail below) was based on a psychometric function having the mathematical form of the Weibull function:

$$
P(X)=1.0-\left[(1.0-\gamma)^{*} e^{-(X / \alpha)^{\alpha}}\right],
$$

where $X$ is the stimulus contrast, $P(X)$ is the probability of making a correct response with stimulus $\mathrm{X}, \alpha$ is the threshold contrast (that giving a correct response probability of 0.82 ), $\beta$ is the slope of the psychometric function (a value of 3.5 was used in these measurements), and $\gamma$ is the probability of a correct response by chance alone (a value of 0.5 was used here, as is appropriate with the $2 \mathrm{AFC}$ paradigm). The Weibull function is commonly used as the mathematical representation of the psychometric function. Some of its properties have been discussed by Nachmias (1981).

A computer-controlled adaptive procedure based on a maximum likelihood estimation of the parameter $\alpha$ was used to find the threshold contrast for each prototype-phase-distorted stimulus pair. The principles of this method, called PEST, have been described by Pentland (1980) and Watson and Pelli (1983). The PEST procedure was implemented as a package of FORTRAN subroutines for the DEC LSI-11/23 by the first author and are available from him. The PEST procedure varies the stimulus contrast from trial to trial in order to find the contrast corresponding to threshold discrimination performance of 0.82 probability correct. The $95 \%$ statistical confidence interval of this estimated threshold contrast was set by the experimenters at $0.15 \log$ units. We can therefore be highly confident that the actual threshold contrast values lie within $0.3 \mathrm{log}$ units of the measured values.

In the experimental trials, the subject was shown two stimuli sequentially. One of these was the original pattern and one was a phasedistorted version. The subject's task was to judge which stimulus, the first or the second, was the original, undistorted stimulus. Depending on the correctness of the subject's forced-choice response, the computer program raised or lowered the contrast of the two stimuli for the next trial. These trials continued until the threshold contrast had been estimated to the specified degree of accuracy. Typically, between 20 and 40 trials were required for each stimulus pair.

The stimuli were presented centered on two different loci within the visual field: the fovea $\left(0^{\circ}\right)$ and $2^{\circ}$ in the right visual field. The foveal stimuli were tested with two different exposure durations: $1,000 \mathrm{msec}$ and $125 \mathrm{msec}$. The $2^{\circ}$ peripheral stimuli were presented only for $1,000 \mathrm{msec}$ because the task was not possible to perform at $125 \mathrm{msec}$.

\section{RESULTS}

The results are first presented in Figures 1-4, in which the stimulus contrast for threshold discrimination is plotted as a function of the six levels of phase distortion. The main features of these graphs are seen in Figure 1, which presents the mean foveal data of the two observers for the two exposure durations. The subjects' individual data were very similar, and no distortion of the data is caused

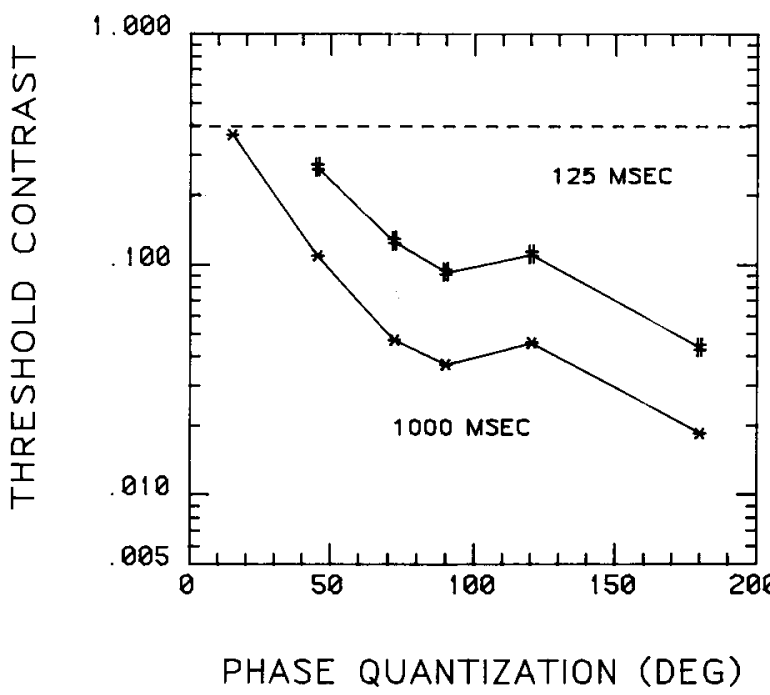

Figure 1. Mean threshold contrast for phase-distortion discrimination as a function of size of phase distortion, for two exposure durations with foveal viewing. The data described by * were measured at an exposure duration of $1,000 \mathrm{msec}$; those described by \# were measured at 125 msec. Each curve is the mean of data from the four separate spatial frequency bands and from two observers. The horizontal dashed line indicates the highest stimulus contrast possible; thresholds at this level represent no discrimination. 
by averaging them together. At the lowest level of phase distortion, $15^{\circ}$ phase quantization, the subjects were unable to discriminate the distorted stimulus from the original (the dashed line represents the highest level of contrast possible with this equipment). As the phase distortion increased, the subjects required less and less stimulus contrast in order to discriminate the distorted stimulus from the original. Figure 1 also shows the effect of exposure duration on phase discrimination: The subjects were more sensitive (required less contrast) with an exposure duration of $1,000 \mathrm{msec}$ than with one of $125 \mathrm{msec}$. This effect of exposure duration is seen at all phase-distortion levels tested, except in those cases where discrimination was not possible (e.g., at $15^{\circ}$ quantization steps).

The effect of phase distortion in different spatial frequency bands is shown in Figures 2 and 3 for the foveal viewing condition, for exposure durations of $1,000 \mathrm{msec}$ (Figure 2) and $125 \mathrm{msec}$ (Figure 3). The main features of Figure 1 are repeated in Figures 2 and 3. At the lowest level of phase distortion, discrimination was not possible even at the highest contrast. At higher distortion levels, however, discrimination was easily performed, with the threshold contrast for discrimination an inverse function of the amount of phase distortion. In both figures, one can see that discrimination performance is about equal for the spatial frequency bands of 2,4 , and $8 \mathrm{cpd}$, but becomes somewhat worse (i.e., requires higher levels of stimulus contrast) for the 16-cpd band.

Figure 4 presents the data for discrimination when the patterns were presented $2^{\circ}$ in the right visual field for an exposure duration of $1,000 \mathrm{msec}$. A comparison of Figure 4 with Figure 2 shows that discrimination at $2^{\circ}$

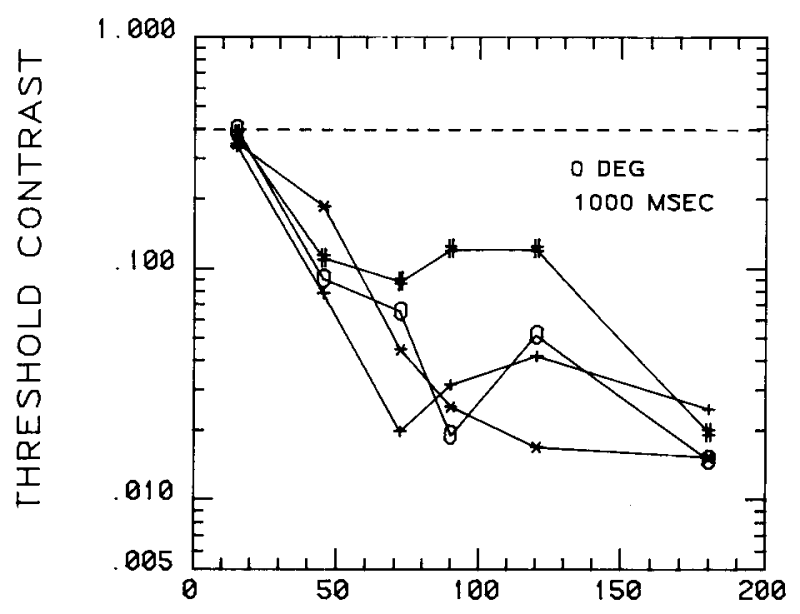

\section{PHASE QUANTIZATION (DEG)}

Figure 2. Threshold contrast for pattern discrimination as a function of size of phase-quantization distortion for an exposure duration of 1,000 msec for foveal viewing, averaged across the two observers. The four curves represent stimuli containing two-octave-wide bands centered on the following spatial frequencies: $*=2 \mathrm{cpd}$; + $=4 \mathrm{cpd} ; 0$ = $8 \mathrm{cpd}$; $\#=16 \mathrm{cpd}$.

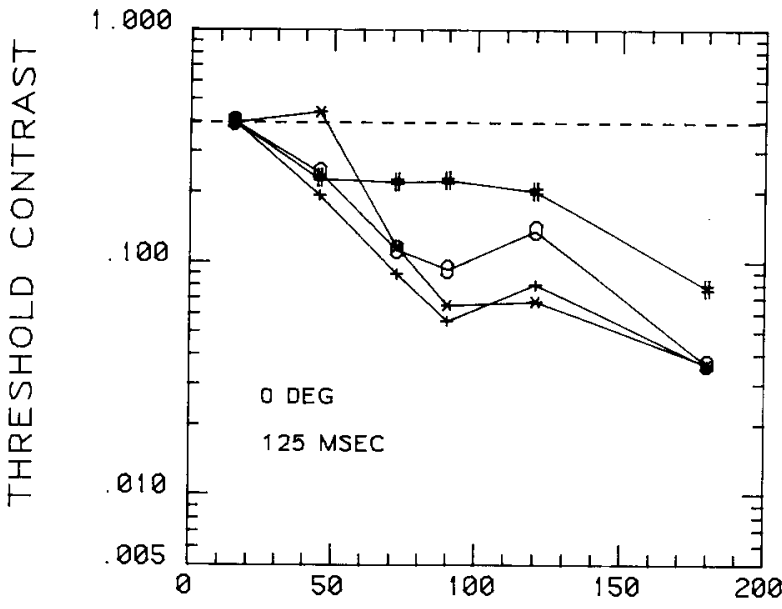

\section{PHASE QUANTIZATION（DEG）}

Figure 3. Threshold contrast for pattern discrimination as a function of size of phase-quantization distortion for an exposure duration of $125 \mathrm{msec}$ for foveal viewing, averaged across the two observers. The four curves represent stimuli containing two-octave-wide bands centered on the following spatial frequencies: $*=2 \mathrm{cpd} ;+$ $=4 \mathrm{cpd} ; 0=8 \mathrm{cpd} ; \#=16 \mathrm{cpd}$.

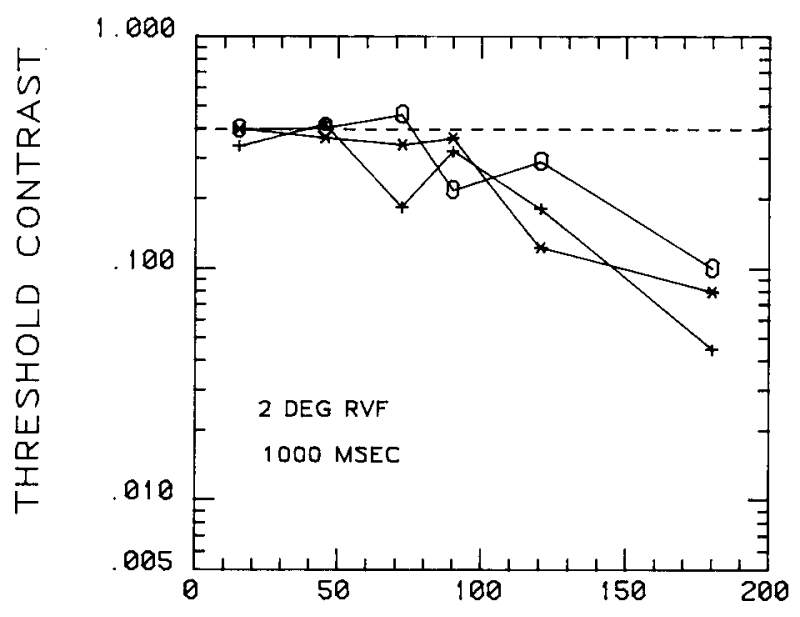

PHASE QUANTIZATION (DEG)

Figure 4. Threshoid contrast for pattern discrimination as a function of size of phase-quantization distortion for an exposure duration of 1,000 msec for retinal eccentricity of $2^{\circ}$ in the right visual field, averaged across the two observers. The three curves represent stimuli containing two-octave-wide bands centered on the following spatial frequencies: $*=2 \mathrm{cpd} ;+=4 \mathrm{cpd} ; 0=8 \mathrm{cpd}$.

eccentricity requires more contrast than that required for the corresponding conditions of foveal viewing.

Another way to illustrate the effect of retinal eccentricity, exposure duration, and spatial frequency band is to represent each of the curves in Figures 2, 3, and 4 by a single number: the amount of phase distortion that would have made the discrimination threshold occur at a stimu- 
lus contrast of 0.10 . This measure was obtained by fitting an exponential function to each set of data shown in Figures 2, 3, and 4, using the least squares criterion. Each best-fitting equation was then used to find the value of phase quantization that would have given a threshold contrast of 0.10 . Although it is not quite accurate to do so, we will refer to this phase angle as "threshold phase."

Threshold phase as a function of spatial frequency band for foveal and $2^{\circ}$ viewing conditions is presented in Figure 5. We can interpret a lower phase threshold as representing a higher sensitivity to phase distortion. The three viewing conditions shown in Figure 5 share common features: A longer exposure duration gives higher phase sensitivity, phase sensitivity is considerably higher for foveal viewing than for peripheral viewing, and the highest phase sensitivity occurs in the 4-cpd band and is worse for both lower and higher frequencies, especially for the two-octave band centered on $16 \mathrm{cpd}$.

\section{DISCUSSION}

Our major finding is the pronounced loss of sensitivity to phase quantization with $2^{\circ}$ peripheral viewing, compared with foveal viewing. The threshold phase distortion in the spatial frequency band centered at $2 \mathrm{cpd}$ was $32^{\circ}$ for foveal viewing with the 1,000 -msec exposure duration (see Figure 5), a result consistent with the measurements of both Burr (1980) and Caelli and Bevan (1982). This threshold rises from $32^{\circ}$ to $123^{\circ}$ when the 1,000 -msec stimuli are viewed $2^{\circ}$ in the peripheral visual field (Figure 5), an increase by a factor of 3.79. The extrafoveal elevation of the phase threshold is 3.84 and 3.85 in the bands centered at $4 \mathrm{cpd}$ and $8 \mathrm{cpd}$, as shown in Ta-

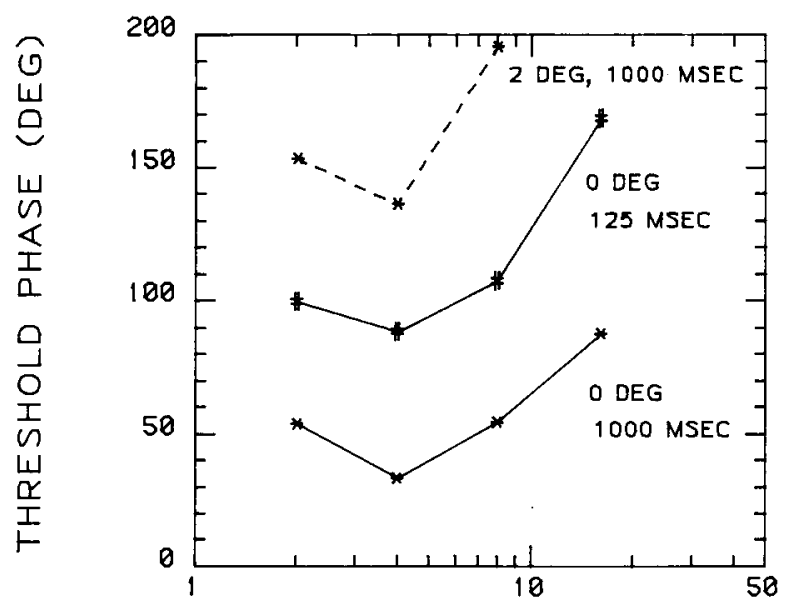

\section{CENTER FREQUENCY (CPD)}

Figure 5. Amount of phase distortion required for discrimination threshold of 0.10 contrast at 0.82 probability of correct response as a function of spatial frequency band center. Solid lines are for foveal viewing; dashed line is for $2^{\circ}$ eccentricity in the right visual field; * is for 1,000-msec exposure duration; \# is for 125 -msec exposure duration.
Table 1

Foveal and extrafoveal thresholds for grating detection and phase resolution in checkerboard patterns.

\begin{tabular}{cccccccc}
\hline & \multicolumn{3}{c}{ Contrast Threshold } & & \multicolumn{3}{c}{ Phase Resolution } \\
\cline { 2 - 4 } \cline { 6 - 8 } Spatial Frequency & $0^{\circ}$ & $2^{\circ}$ & Elevation & & $0^{\circ}$ & $2^{\circ}$ & Elevation \\
\hline 2 cpd & 0.013 & 0.020 & 1.54 & & 38 & 144 & 3.79 \\
4 cpd & 0.008 & 0.016 & 2.00 & & 32 & 123 & 3.84 \\
8 cpd & 0.013 & 0.030 & 2.31 & & 40 & 154 & 3.85 \\
\hline
\end{tabular}

The grating data are for a circular field $3^{\circ}$ in diameter (from Hilz et al., 1981 , Figure 1b). Phase resolution data are the data shown in Figure 5 of the present study. Note that in the latter case, the spatial frequency figure refers to the center frequency of a two-octave-wide band.

ble 1 . On the other hand, the elevation of the detection thresholds for sinusoidal gratings of 2,4 , and $8 \mathrm{cpd}$ is $2-, 1.6-$, and 3 -fold, respectively, at $2^{\circ}$ retinal eccentricity (see Table 1). We can be confident that the reduced sensitivity to phase quantization in extrafoveal vision is not simply a consequence of reduced contrast sensitivity to global spatial frequency components of the target patterns.

At the brief exposure duration $(125 \mathrm{msec})$ and $2^{\circ}$ retinal eccentricity, neither of the subjects could discriminate the original texture from any of its phase-quantized versions, even at the highest possible contrast. In a previous study, we obtained virtually the same result for the affected eyes of both strabismic and anisometropic amblyopes (Weiss, Rentschler, \& Caelli, 1985). Earlier findings by Hess, Campbell, and Zimmern (1980) lead us to conclude that such phase resolution deficits arise from a specific loss of foveal visual function. The present finding of a lack of phase resolution in rapid extrafoveal pattern discrimination supports this notion.

From the data presented in Figure 5, it seems that under the conditions tested, phase resolution is highest in the spatial frequency band centered on $4 \mathrm{cpd}$, extending from 2 to $8 \mathrm{cpd}$. These data do not, however, show that the observed superiority of phase resolution is a result of the specific spatial frequency band considered. Caelli and Bevan (1982) have emphasized that the amplitude of each spatial frequency component determines the detectability of its phase value. The energy present in each band needs to be considered before conclusions can be drawn as to the spatial frequency dependence of phase effects. In the case of the texture used in the present study, the percentage of amplitude components in the band centered at $4 \mathrm{cpd}$ is higher than in the other bands (Caelli \& Bevan, 1982, Figure 4a, Texture $T_{3}$ ). We conclude that our results are consistent with Caelli and Bevan's finding of an independence of phase resolution from spatial frequency.

Although we have shown that sensitivity to phase distortion in peripheral vision cannot be explained solely in terms of reduced grating contrast sensitivity, our results do not imply an insensitivity of peripheral vision to phase information per se. Rentschler and Treutwein (1985) have shown that two different mechanisms are involved in the discrimination of compound gratings with identical power spectra. Gratings that differ in spatial contrast densities 
(i.e., in first-order joint probability density) are distinguished on the basis of local energy detection. This mechanism ignores the spatial relationships of points within an image. Gratings that have identical second-order statistics are distinguished by means of a mechanism that registers the positional relationships of image components. Neither of these mechanisms analyzes global Fourier phase, but both act on specific properties of image luminance profiles.

The form of phase modulation used in the present study does change the local densities of contrast in the textures (i.e., their first-order statistics). We can assume that the resulting image distortions are detected on the basis of local processing of luminance profile characteristics. The use of the phase-quantization paradigm does not, therefore, test the sensitivity of "certain phase selective devices" (Atkinson \& Campbell, 1974) in peripheral vision. Rather, this technique provides a powerful tool for the investigation of peripheral visual sensitivity to image structure that is not captured by measuring conventional grating detectability. It should be used to further probe these characteristics of human vision.

\section{REFERENCES}

Atrinson, J., \& Campbell, F. W. (1974). The effect of phase on the perception of compound gratings. Vision Research, 14, 159-162.

AUBERT, H., \& FörSTER, R. (1857). Beitrage zur Kenntnis des indirekten Sehens. I. Über den Raumsinn der Retina. Albert von Graefes Archiv fur klinische und experimentelle Ophthalmologie, III, 1-37.

AULHORN, E., \& HARMS, H. (1972). Visual perimetry. In D. Jameson \& L. M. Hurvich (Eds.), Handbook of sensory physiology, Vol. VII/4: Visual psychophysics (pp. 102-145). New York: Springer.

BRADDICK, O. (1981). Is spatial phase degraded in peripheral vision and visual pathology? Documenta Ophthalmologica, Proceedings Series $30,255-262$.

Brettel, H., Caelli, T., Hilz, R., \& Rentschler, I. (1982). Modelling perceptual distortion: Amplitude and phase processing in the human visual system. Human Neurobiology, 1, 61-67.

Brigham, E. O. (1974). The fast fourier transform. Englewood Cliffs, NJ: Prentice-Hall.

BURR, D. (1980). Sensitivity to spatial phase. Vision Research, 20 , 391-396.

Caeld, T., \& Bevan, P. (1982). Visual sensitivity to two-dimensional spatial phase. Journal of the Optical Society of America, 72, 1375-1381.

DaITCH, J. M., \& GREen, D. G. (1969). Contrast sensitivity of the human peripheral retina. Vision Research, 9, 947-952.

GrahaM, N. (1972). Spatial frequency channels in the human visual system: Effects of luminance and pattern drift rate. Vision Research, $12,53-68$.
Graham, N., \& Nachmias, J. (1971). Detection of grating patterns containing two spatial frequencies: A comparison of single-channel and multiple-channel models. Vision Research, 11, 251-259.

Harvey, L. O., Jr., \& PöpPel, E. (1972). Contrast sensitivity of the human retina. American Journal of Optometry \& Archives of American Academy of Optometry, 49, 748-753.

Hess, R. F., CAMpbell, F. W., \& Zimmern, R. (1980). Differences in the neural basis of human amblyopias: The effect of mean luminance. Vision Research, 20, 295-305.

Hilz, R., \& Cavonius, C. R. (1974). Functional organization of the peripheral retina: Sensitivity to periodic stimuli. Vision Research, 14, 1333-1337.

Hilz, R., Rentschler, I., \& Brettel, H. (1981). Insensitivity of peripheral vision to spatial phase. Experimental Brain Research, 43, $111-114$.

JuLEsz, B. (1981). Textons, the elements of texture perception, and their interactions. Nature, 290, 91-97.

KELLY, D. H. (1984a). Retinal inhomogeneity: I. Spatiotemporal contrast sensitivity. Journal of the Optical Society of America A, 1, 107-113.

KeLLY, D. H. (1984b). Retinal inhomogeneity: II. Spatial summation. Journal of the Optical Society of America A, 1, 114-119.

LAWDEN, M. C. (1983). An investigation of the ability of the human visual system to encode spatial phase relationships. Vision Research, 23, 1451-1463.

NaChmias, J. (1981). On the psychometric function for contrast detection. Vision Research, 21, 215-223.

NAChmiAs, J., \& WeBer, A. (1975). Discrimination of simple and complex gratings. Vision Research, 15, 217-223.

OPPENHEIM, A. V., \& LiM, J. S. (1981). The importance of phase in signals. Proceedings of the IEEE, 69, 529-541.

Pentland, A. (1980). Maximum likelihood estimation: The best PEST Perception \& Psychophysics, 28, 377-379.

Pöppel, E., \& Harvey, L. O., JR. (1973). Light-difference threshold and subjective brightness in the periphery of the visual field. Psychologische Forschung, 36, 145-161.

Rentschler, I., \& Treutwein, B. (1985). Loss of spatial phase relationships in extrafoveal vision. Nature, 313, 308-310.

Rovamo, J., \& Virsu, V. (1979). An estimation and application of the human cortical magnification factor. Experimental Brain Research, 37, 495-510.

Virsu, V., \& Rovamo, J. (1979). Visual resolution, contrast sensitivity and the cortical magnification factor. Experimental Brain Research, 37, 475-494.

WAtson, A. B., \& Pelli, D. G. (1983). QUEST: A Bayesian adaptive psychometric method. Perception \& Psychophysics, 33, 113-120.

Weiss, C., Rentschler, I., \& CAELl, T. (1985). Amblyopic processing of positional information. Part II: Sensitivity to phase distortion. $E x$ perimental Brain Research, 60, 279-288.

WERTHEIM, T. (1894). Uber die indirekte Sehschärfe. Zeitschrift für Psychologie und Physiologie der Sinnesorgane, 7, 172-187.

(Manuscript received April 8, 1985; revision accepted for publication October 14, 1985.) 